\title{
Percutaneous Transsplenic Balloon-Assisted Transjugular Intrahepatic Portosystemic Shunt Placement in Patients with Portal Vein Obliteration for Portal Vein Recanalization: Feasibility, Safety and Effectiveness
}

\author{
T. C. Meine ${ }^{1}$ - L. S. Becker ${ }^{1}$ C. L. A. Dewald ${ }^{1}$ - S. K. Maschke ${ }^{1}$ B. Maasoumy ${ }^{2}$ • \\ E. Jaeckel $^{2} \cdot$ H. Wedemeyer ${ }^{2}$ - F. K. Wacker ${ }^{1}$ B. C. Meyer ${ }^{1}$ - J. B. Hinrichs ${ }^{1}$ (i)
}

Received: 7 September 2021 / Accepted: 30 December 2021/Published online: 11 January 2022

(C) The Author(s) 2022

\begin{abstract}
Purpose To assess the feasibility, safety and effectiveness of portal vein recanalization (PVR)-transjugular portosystemic shunt (TIPS) placement via splenic access using a balloon puncture technique.

Materials and Methods In a single-center retrospective study from March 2017 to February 2021, 14 consecutive patients with portal hypertension, chronic liver disease and portal vein occlusion or near-complete ( $>95 \%)$ occlusion were referred for PVR-TIPS placement. Feasibility, safety and effectiveness including procedural characteristics such as technical success, complication profile and splenic access time (SAT), balloon positioning time (BPT), conventional portal vein entry time (CPVET), overall procedure time (OPT), fluoroscopy time (FT), dose-area product (DAP) and air kerma (AK) were evaluated.
\end{abstract}

B. C. Meyer and J. B. Hinrichs are contributed equally in this work.

\footnotetext{
J. B. Hinrichs

Hinrichs.jan@mh-hannover.de

T. C. Meine

meine.timo@mh-hannover.de

L. S. Becker

becker.lena@mh-hannover.de

C. L. A. Dewald

dewald.cornelia@mh-hannover.de

S. K. Maschke

maschke.sabine@mh-hannover.de

B. Maasoumy

Maasoumy.Benjamin@mh-hannover.de

E. Jaeckel

Jaeckel.Elmar@mh-hannover.de
}

Results Transsplenic PVR-TIPS using balloon puncture technique was technically feasible in 12 of 14 patients ( 8 men, $49 \pm 13$ years). In two patients without detectable intrahepatic portal vein branches, TIPS placement was not feasible and both patients were referred for further treatment with nonselective beta blockers and endoscopic variceal ligation. No complications grade $>3$ of the Cardiovascular and Interventional Radiological Society of Europe classification system occurred. The SAT was $25 \pm 21 \mathrm{~min}$, CPVET was $33 \pm 26 \mathrm{~min}$, the OPT was $158 \pm 54 \mathrm{~min}$, the FT was $42 \pm 22 \mathrm{~min}$, the DAP was $167.84 \pm 129.23 \mathrm{~Gy}^{*} \mathrm{~cm}^{2}$ and the AK was $1150.70 \pm 910.73 \mathrm{mGy}$.

Conclusions Transsplenic PVR-TIPS using a balloon puncture technique is feasible and appears to be safe in our series of patients with obliteration of the portal vein. It expands the interventional options in patients with chronic PVT.

\author{
H. Wedemeyer \\ Wedemeyer.Heiner@mh-hannover.de \\ F. K. Wacker \\ wacker.frank@mh-hannover.de \\ B. C. Meyer \\ meyer.bernhard@mh-hannover.de \\ Institute for Diagnostic and Interventional Radiology, \\ Hannover Medical School, OE8220 Carl-Neuberg-Straße 1, \\ 30625 Hannover, Germany \\ 2 Department of Hepatology, Gastroenterology and \\ Endocrinology, Hannover Medical School, Carl-Neuberg- \\ Straße 1, 30625 Hannover, Germany
}


Keywords Portal vein recanalization-Transjugular intrahepatic portosystemic shunt · Portal vein obliteration - Portal vein thrombosis - Transsplenic . Splenic access $\cdot$ Balloon puncture technique

$\begin{array}{ll}\text { Abbreviations } \\ \text { AK } & \text { Air Kerma } \\ \text { BPT } & \text { Balloon Positioning Time } \\ \text { CIRSE } & \begin{array}{l}\text { Cardiovascular and Interventional Radiological } \\ \text { Society of Europe }\end{array} \\ \text { CPVET } & \text { Conventional Portal Vein Entry Time } \\ \text { DAP } & \text { Dose-Area Product } \\ \text { FT } & \text { Fluoroscopy Time } \\ \text { MELD } & \text { Model of End-Stage Liver Disease } \\ \text { OPT } & \text { Overall Procedural Time } \\ \text { PV } & \text { Portal Vein } \\ \text { PVO } & \text { Portal Vein Obliteration } \\ \text { PVR } & \text { Portal Vein Recanalization } \\ \text { PVT } & \text { Portal Vein Thrombosis } \\ \text { SAT } & \text { Splenic Access Time } \\ \text { SV } & \text { Splenic Vein } \\ \text { TIPS } & \text { Transjugular Intrahepatic Portosystemic Shunt }\end{array}$

\section{Introduction}

Transjugular intrahepatic portosystemic shunt (TIPS) is an effective intervention to decompress portal hypertension and is recommended in patients with refractory ascites or gastrointestinal bleeding [1]. Portal hypertension due to portal vein thrombosis (PVT) might result in chronic obliteration of the portal vein (PVO), which causes morbidity and preclude patients from liver transplantation $[2,3]$. TIPS creation is a complex procedure with high failure and complication rates in patients with PVO [2]. To overcome these challenges, recanalization of the portal vein (PVR) in combination with TIPS placement has been proposed [4]. Case series data suggest PVR-TIPS via splenic access has a high shunt patency, improves the survival of the patients and enables portal vein (PV) anastomosis in transplant candidates with chronic PVT [3-5]. However, there are a limited number of publications addressing technical aspects of the procedure $[2,4,6]$. We therefore sought to evaluate the feasibility, safety and efficacy of PVR-TIPS using a splenic access and a balloon puncture technique assessing the technical success, complication profile and peri-procedural characteristics.
Materials and Methods

\section{Study Population}

Between March 2017 and February 2021, 209 TIPS procedures were performed at our tertiary referral center and were retrospectively reviewed. Overall, 196 TIPS placements via a transjugular access were successful, which is also our standard approach in patients with PVT. In this period, 14 patients presented with chronic PVO defined as $>95 \%$ occlusion of the PV with or without portal cavernoma and PVT $>27$ days after onset of symptoms $[3,7]$. All patients underwent a standardized pre- and postinterventional workflow as described before and were included in the study [8].

\section{Transsplenic PVR-TIPS}

All transsplenic PVR-TIPS procedures were performed under general anesthesia by board-certified interventional radiologists (J.B.H., B.C.M.). First, a peripheral branch of the splenic vein (SV) was percutaneously punctured under ultrasound guidance with a micro-puncture set (4-F Custom Procedure Kit, Merit Medical). After successful SV access, a 4-F micro-puncture sheath was introduced. A 0.035 -inch guidewire $(150 / 260 \mathrm{~cm}$, Terumo Corporation) was advanced and a 4-F sheath (Avanti ${ }^{\circledR}+$, Cordis) was placed in the SV. Then, a 4-F diagnostic catheter (Berenstein, Impress, Merit Medical) was introduced in the SV and a splenoportogram was acquired with manual contrast injection to detect any residual PV branches (Fig. 1). The diagnostic catheter was gently advanced with the guidewire through the occluded portion of the PV or through a filiform portal cavernoma branch connected into an intrahepatic PV branch (Fig. 2). In this position, a balloon catheter $(5 / 6 \mathrm{mmx} 40 \mathrm{~mm}, 135 \mathrm{~cm}$, Sterling, Boston Scientific) was inflated as fluoroscopic target. Afterward, the TIPS needle (GORE TIPS Set, W.L.Gore\&Associates) was advanced through a standard transjugular access into an appropriate hepatic vein and the balloon-dilated PV branch was punctured under fluoroscopy (Fig. 2). In case of successful puncture of the balloon cover, a 0.014-inch wire (V-14, Control Wire, Boston Scientific) was introduced through the TIPS needle and captured at the splenic access site. When the TIPS needle entered the PV branch without perforating the balloon cover, this was verified by aspiration of blood and injection of contrast media. Finally, a 0.035 -inch guidewire was introduced through the transjugular access in the superior mesenteric vein or SV and a standard TIPS placement with a TIPS stent graft (GORE Viatorr TIPS Endoprosthesis with controlled Expansion, W.L.Gore\&Associates) was performed (Fig. 2) [8]. 


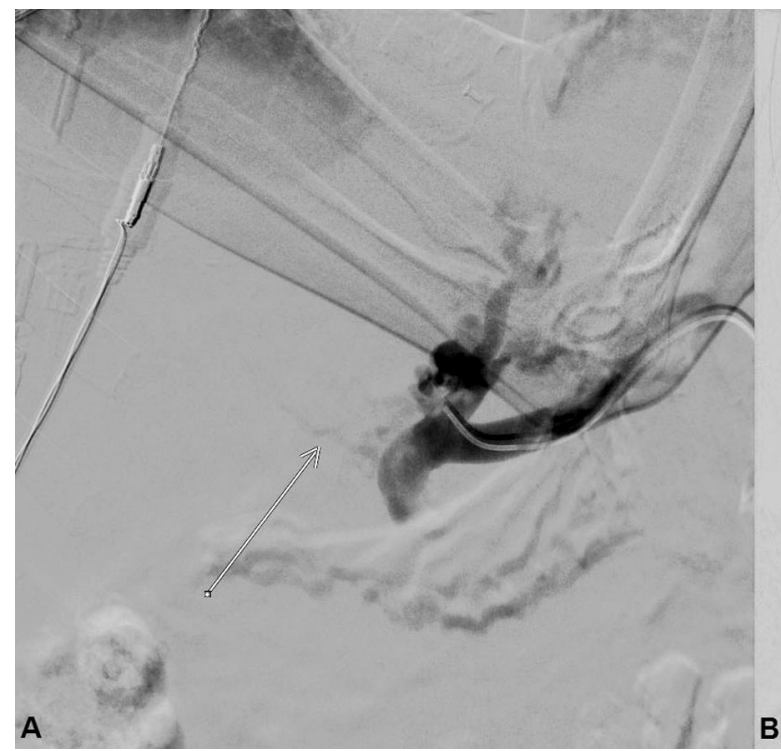

Fig. 1 Splenoportogram of portal vein obliteration. A This splenoportogram was generated via a diagnostic catheter in the splenic vein. It shows the occlusion $>95 \%$ of the portal vein with a filiform residual portal vein branch (white arrow). Collateralization of the

\section{Data Collection and Statistical Analysis}

Patient demographics and procedural characteristics including the complication profile according the Cardiovascular and Interventional Radiological Society of Europe (CIRSE) classification system were obtained from medical reports $[8,9]$. The overall procedural time (OPT), fluoroscopy time (FT) and the dose-area product (DAP) were determined as defined elsewhere [8]. Furthermore, the splenic access time (SAT) as the puncture time of the SV confirmed by contrast media or an established guidewire in the SV branch and the balloon positioning time (BPT) as following the time until inflation of the balloon catheter as fluoroscopic target were assessed from interventional imaging. In addition, conventional portal vein entry time (CPVET) was determined as the time from the catheterized hepatic vein to the first documented image with the wire in the balloon cover or dilated PV branch. Finally, air kerma (AK) was recorded to evaluate the radiation exposure. Values are shown as mean \pm standard deviation.

\section{Results}

Patients' demographics are described in detail in Table 1, and the procedural characteristics are given in Table 2 and Supplements. The transsplenic PVR-TIPS via balloon puncture technique was technically successful in 12 of 14 patients. In two patients with portal cavernoma, no intrahepatic PV branch could be detected at the time of

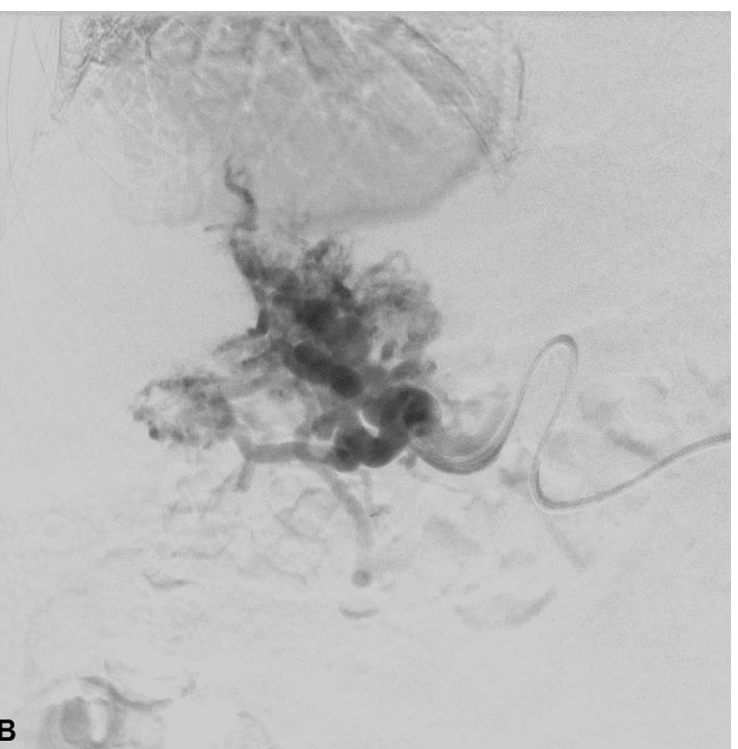

occlusion is conducted via esophagogastric varices. B In this digital subtraction angiography acquired via the splenic vein/portal confluence, a complete occlusion of the portal vein with cavernous transformation is present

intervention and the procedure was aborted. Both patients were referred for further treatment with nonselective beta blockers and/or endoscopic variceal ligation and are under surveillance. No complication grade $>3$ of the CIRSE classification system occurred. Grade 3 complications were treated with blood transfusion (one subcapsular splenic hematoma), antibiotic medication (one spontaneous bacterial peritonitis) and compression (two secondary access site bleedings).

\section{Discussion}

PVR-TIPS placement using a splenic access has been shown to improve survival and transplant candidacy in patients with chronic PVT [5], but it is still challenging due to the lack of PV target. Fluoroscopic targets within the obliterated PV branch have been reported to be useful $[3,4]$. Habib et al. advanced a snare device through a splenic access in the PV branch to guide the intrahepatic PV puncture [3]. In contrast, Chen et al. used a balloon catheter as fluoroscopic target in the PV branch via a transhepatic approach [6]. We combined both approaches using a splenic access and a balloon catheter to assist PVRTIPS placement, because the balloon catheter might serve as a larger fluoroscopic target in an obliterated PV branch as the snare device might not fully deploy.

Procedural characteristics for transsplenic PVR-TIPS are only available from Habib's study [4]. The technical success rate in our study with $85 \%$ was slightly inferior to 


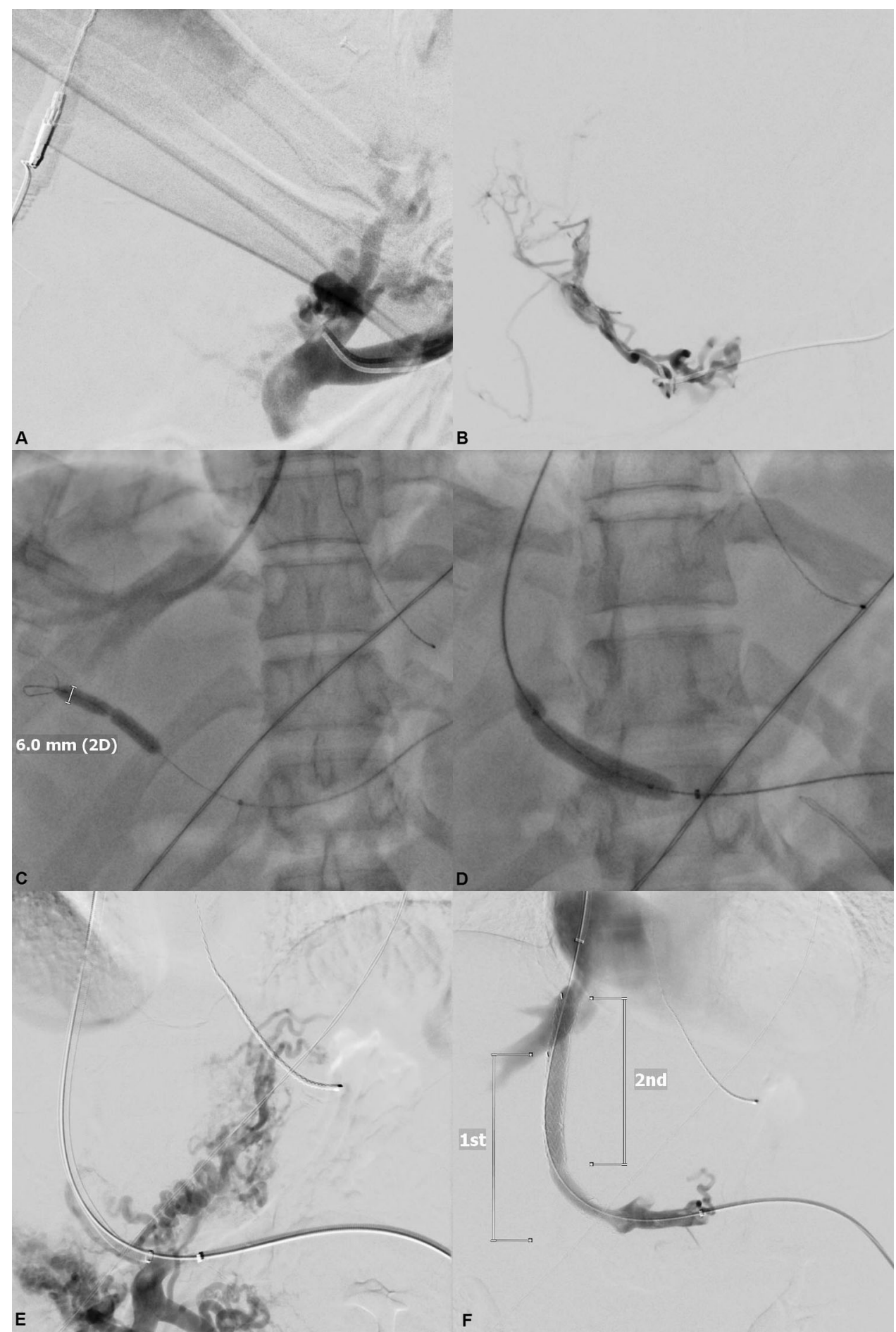

Fig. 2 Balloon puncture technique for transsplenic portal vein recanalization-transjugular intrahepatic portosystemic shunt placement. A After successful splenic access was achieved, a 4-F diagnostic catheter was introduced in the splenic vein and a splenoportography the occlusion of $>95 \%$ of the portal vein was confirmed. B The obliterated portal vein was gently catheterized with a micro-catheter in this case and filiform original intrahepatic portal vein branches could be detected. C After recanalization of the portal vein, a 6-mm balloon catheter was inflated as the fluoroscopic target in the intrahepatic portal vein branch. The TIPS needle was introduced through a standard transjugular access into the appropriate hepatic vein. D After successful intrahepatic puncture under fluoroscopy guidance from the hepatic vein into the balloon-dilated portal vein branch, a control wire was introduced via the TIPS needle and is captured at the splenic access site. Then, a standard TIPS procedure was performed with gently dilatation of the puncture tract. E The TIPS sheath was advanced in through the TIPS tract and a portography was generated to evaluate the position of the TIPS stent graft. F A final portography was generated to confirm sufficient flow through the TIPS stent graft. In this case, a second TIPS stent graft was placed to cover the complete length of the TIPS tract 
Table 1 Patient's characteristics

\begin{tabular}{ll}
\hline Number & 14 \\
Age (years) & $49 \pm 13$ \\
Sex (male/female) & $8 / 6$ \\
MELD score & $10 \pm 6$ \\
Liver disease & \\
Alcohol-induced & 5 \\
HBV & 1 \\
AIH & 2 \\
PSC & 1 \\
SSC & 1 \\
FNH/Adenoma & 2 \\
Congenital fibrosis & 1 \\
Cryptogenic & 1 \\
Complications of portal hypertension & 10 \\
Refractory variceal or gastrointestinal bleeding & 4 \\
Refractory ascites and hydrothorax & 8 \\
Portal cavernoma &
\end{tabular}

Age, sex, model of end-stage liver disease (MELD) score, liver disease and complication of portal hypertension are tabulated for the study population. One patient with focal nodular hyperplasia had essential thrombocytosis as another cause for portal hypertension

$H B V$ hepatitis virus $\mathrm{B}, A I H$ autoimmune hepatitis, $P S C$ primary sclerosing cholangitis, SSC secondary sclerosing cholangitis, FNH focal nodular hyperplasia
Habib et al. with $100 \%$ [3]. In two cases in our study, no intrahepatic PV branch could be identified at the time of the intervention. The final interventional option in these patients might be the creation of an extrahepatic or extracorporeal portosystemic shunt, e.g., via a transmesenteric or direct percutaneous access [10-12].

No major complications occurred in our study. The reported complications in our study required no further interventional or surgical treatment, which reflects the safety of our technique.

The effectiveness of the balloon puncture technique can be analyzed using the FT [13]. The mean FT of $42 \mathrm{~min}$ in our study is even lower than the reported FT of $53 \mathrm{~min}$ for the snare puncture technique although the group from Chicago did not perform variceal embolization [3]. The dilatation of the PV branch with the balloon as a fluoroscopic target offers visual and haptic feedback that might accelerate the puncture compared to the snare puncture technique.

Considering the procedural times, the mean OPT of $158 \mathrm{~min}$ of our transsplenic balloon-assisted PVR-TIPS procedure is comparable to the snare puncture technique with a mean procedural time of $147 \mathrm{~min}$ [3]. Time-consuming steps include splenic access and probing the obliterated PV branch. Catheterization of the SV and dilatation of the intrahepatic PV branch account for $30 \%$ of
Table 2 Procedural characteristics

\begin{tabular}{ll}
\hline Technical success & $12 / 14$ \\
Additional procedures & \\
Second stent-graft placement & 6 \\
Variceal embolization & 4 \\
Complications according CIRSE classification system & $7 / 14$ \\
1 & $0 / 14$ \\
2 & $3 / 14$ \\
3 & $0 / 14$ \\
4 & $0 / 14$ \\
5 & $0 / 14$ \\
6 & \\
Procedural times & $25 \pm 21$ \\
Splenic access time (min) & $55 \pm 35$ \\
Balloon positioning time (min) & $33 \pm 26$ \\
Conventional portal vein entry time (min) & $158 \pm 54$ \\
Overall procedural time (min) & \\
Radiation exposure & $42 \pm 22$ \\
Fluoroscopy time (min) & $167.84 \pm 129.23$ \\
Dose-area product (Gy*cm2) & $1150.70 \pm 910.73$ \\
Air kerma (mGy) & \\
\hline Procedural characterics for the study populat
\end{tabular}

Procedural characteristics for the study populations included technical success rate, additional procedures, complications, procedural times and radiation exposure. In one patient, the balloon puncture time could not be determined due to lack of data

CIRSE Cardiovascular and Interventional Radiological Society of Europe 
the OPT. Finally, the relatively high radiation exposure in our study is still below the TIPS reference level [14]. Focused on the procedural characteristics, the SAT was shorter than the BPT, but the CPVET was not increased in relation to the literature [8]. OPT, FT, DAP and AK were comparably high for TIPS procedures [8].

Small sample size and retrospective study design are study limitations. Furthermore, no follow-up data for this cohort were assessed pertaining clinical outcomes and patency rates.

\section{Conclusion}

Overall, transsplenic PVR-TIPS using a balloon puncture technique was technically feasible and appears to be safe. It is an alternative to the reported snare puncture technique in patients with chronic PVT.

Funding Open Access funding enabled and organized by Projekt DEAL. This work was supported by an unrestricted research grant from Siemens Healthcare $\mathrm{GmbH}$ and personal grant practice $\mathrm{MHH}$ DFG-Timo Meine.

\section{Declarations}

Conflict of interest The authors of this manuscript declare relationships with the following companies: Siemens Healthcare and ProMedicus (Bernhard Meyer and Frank Wacker; outside the submitted work). The remaining authors declare no relationships with any companies whose products or services may be related to the subject matter of the article.

Ethical Approval All procedures performed in studies involving human participants were in accordance with the ethical standards of the institutional and/or national research committee and with the 1964 Helsinki Declaration and its later amendments or comparable ethical standards. For this type of study, formal consent is not required. This article does not contain any studies with animals performed by any of the authors.

Consent for Publication Consent for publication was obtained for every individual person's data included in the study.

Supplementary InformationThe online version contains supplementary material available at https://doi.org/10.1007/s00270021-03054-2.

Open Access This article is licensed under a Creative Commons Attribution 4.0 International License, which permits use, sharing, adaptation, distribution and reproduction in any medium or format, as long as you give appropriate credit to the original author(s) and the source, provide a link to the Creative Commons licence, and indicate if changes were made. The images or other third party material in this article are included in the article's Creative Commons licence, unless indicated otherwise in a credit line to the material. If material is not included in the article's Creative Commons licence and your intended use is not permitted by statutory regulation or exceeds the permitted use, you will need to obtain permission directly from the copyright holder. To view a copy of this licence, visit http://creativecommons. org/licenses/by/4.0/.

\section{References}

1. Angeli P, Bernardi M, Villanueva C, Francoz C, Mookerjee RP, Trebicka $J$, et al. EASL Clinical practice guidelines for the management of patients with decompensated cirrhosis. J Hepatol. 2018;69:406-60.

2. Van Ha TG, Hodge J, Funaki B, Lorenz J, Rosenblum J, Straus C, et al. Transjugular intrahepatic portosystemic shunt placement in patients with cirrhosis and concomitant portal vein thrombosis. Cardiovasc Intervent Radiol. 2006;29:785-90.

3. Habib A, Desai K, Hickey R, Thornburg B, Vouche M, Vogelzang RL, et al. Portal vein recanalization-transjugular intrahepatic portosystemic shunt using the transsplenic approach to achieve transplant candidacy in patients with chronic portal vein thrombosis. J Vasc Interv Radiol. 2015;26:499-506.

4. Thornburg B, Desai K, Hickey R, Kulik L, Ganger D, Baker T, et al. Portal vein recanalization and transjugular intrahepatic portosystemic shunt creation for chronic portal vein thrombosis: technical considerations. Tech Vasc Interv Radiol. 2016;19:52-60.

5. Salem R, Vouche M, Baker T, Herrero JI, Caicedo JC, Fryer J, et al. Pretransplant portal vein recanalization-transjugular intrahepatic portosystemic shunt in patients with complete obliterative portal vein thrombosis. Transplantation. 2015;99:2347-55.

6. Chen Y, Ye P, Li Y, Ma S, Zhao J, Zeng Q. Percutaneous transhepatic balloon-assisted transjugular intrahepatic portosystemic shunt for chronic, totally occluded, portal vein thrombosis with symptomatic portal hypertension: procedure technique, safety, and clinical applications. Eur Radiol. 2015;25:3431-7.

7. Rössle M, Bettinger D, Trebicka J, Klinger C, Praktiknjo M, Sturm L, et al. A prospective, multicentre study in acute noncirrhotic, non-malignant portal vein thrombosis: comparison of medical and interventional treatment. Aliment Pharmacol Ther. 2020;52:329-39.

8. Meine TC, Dewald CLA, Becker LS, Mähringer-Kunz A, Massoumy B, Maschke SK, et al. Transjugular intrahepatic portosystemic shunt placement: portal vein puncture guided by $3 \mathrm{D} /$ $2 \mathrm{D}$ image registration of contrast-enhanced multi-detector computed tomography and fluoroscopy. Abdom Radiol (NY). 2020.

9. Filippiadis DK, Binkert C, Pellerin O, Hoffmann RT, Krajina A, Pereira PL. Cirse quality assurance document and standards for classification of complications: the cirse classification system. Cardiovasc Intervent Radiol. 2017;40:1141-6.

10. Yoon JK, Kim M-D, Lee DY, Han SJ. Mesocaval shunt creation for jejunal variceal bleeding with chronic portal vein thrombosis. Yonsei Med J. 2018;59:162.

11. Meyer BC, Hinrichs JB, Ivanyi P, Ehrlich S, Greer M, Hoeper $\mathrm{MM}$, et al. Extracorporeal portosystemic shunt in secondary Budd-Chiari syndrome. J Hepatol. 2020;73:974-6.

12. Entezari P, Riaz A, Thornburg B, Salem R. Percutaneous ultrasound-guided superior and inferior mesenteric vein access for portal vein recanalization-transjugular intrahepatic portosystemic shunt: a case series. Cardiovasc Intervent Radiol. 2021;44:496-9.

13. Marquardt $S$, Rodt $T$, Rosenthal H, Wacker F, Meyer BC. Impact of anatomical, procedural, and operator skill factors on the success and duration of fluoroscopy-guided transjugular intrahepatic portosystemic shunt. Cardiovasc Intervent Radiol. 2015;38:903-12. 
14. Miller DL, Kwon D, Bonavia GH. Reference levels for patient radiation doses in interventional radiology: proposed initial values for U.S. Practice. Radiology. 2009;253:753-64.
Publisher's Note Springer Nature remains neutral with regard to jurisdictional claims in published maps and institutional affiliations. 